\title{
Interface between the intestinal environment and the nervous system
}

\section{O Lundgren}

Possible mechanisms by which the intestinal contents may influence afferent nerve fibres in the lamina propria of the intestinal mucosa are discussed in this brief review. After addressing intestinal epithelial permeability, endocrine and brush cells are discussed, as well as enterocytes, as sensors for luminal microbes.

\section{PERMEABILITY OF THE INTESTINAL EPITHELIUM}

Diffusion of solutes from the gut lumen into the intestinal tissue is dependent on the physicochemical characteristics of the solutes and on the permeability characteristics of the intestinal epithelium. Whereas the intestinal epithelial lining has a high permeability for lipid soluble compounds, the epithelial barrier is much less permeable to water soluble substances. The passive transport (diffusion) of hydrophilic substances across the epithelium occurs via "pores" in the tight junctions between the enterocytes. There are both morphological and functional observations to suggest that a marked difference in tight junction permeability exists between villi and crypts. Freeze fracture techniques have revealed that there are more so-called strands between villous enterocytes than between the enterocytes in the crypts, indicating that the villous epithelium is "tighter" than the crypt epithelium. ${ }^{1}$ This implies that more than $70 \%$ of electrical conductance is attributable to crypts which constitute less than $15 \%$ of the total surface area. Functional studies support this view, suggesting that the absorbing upper parts of the villi contain pores with a radius below $6 \AA$, whereas the pores of crypts are much larger (radius 50-60 ̊) ${ }^{2}$. These values should be compared, for example, with the size of mannitol (molecular mass $180 \mathrm{Da}$ ) which has a molecular radius of approximately $3.5 \AA$. In this context, it should be underlined that the intestinal contents under normal circumstances have an "efficient" contact with only the upper half to two thirds of the villi. ${ }^{3}$ Constant secretion of fluid from the crypts produces a convective flow of fluid that probably markedly slows or prevents solute diffusion into the crypts.

Correspondence to:
Professor O Lundgren Department of Physiology, Sahlgrenska Academy, Göteborg University, Box 432, S-405 30 Göteborg, Sweden; ove.lundgren@ fysiologi.gu.se

In a series of interesting reports, Pappenheimer and colleagues ${ }^{4-6}$ obtained experimental evidence for the view that tight junction permeability is influenced by the intestinal con- example, increases tight junction permeability. Pappenheimer et al proposed, on the basis of their experimental findings, that tight junction pores increased their size whereas other researchers have obtained experimental evidence that the number of, rather than the size of, small pores in villi increase when Na-glucose is transported across the apical membrane of the enterocytes. ${ }^{3}$

To summarise, this brief overview of epithelial permeability suggests that lipid soluble compounds may readily diffuse across enterocytes to influence afferent neurones. Water soluble compounds may cross the epithelial barrier when their molecular mass is below $1 \mathrm{kDa}$. Permeability to hydrophilic compounds may be altered by the transport of luminal nutrients across the apical membrane of enterocytes.

\section{ENDOCRINE CELLS OF THE SMALL INTESTINE}

The gastrointestinal tract is the largest endocrine organ in the body and contains numerous hormones, many of them with as yet unknown physiological functions. The hormones are contained within specialised cells dispersed among the enterocytes. Most of the cells are polarised, with long microvilli at the apical surface projecting into the intestinal lumen. Granules containing 5-hydroxytryptamine (5-HT) and/or peptide hormones are concentrated around the basolateral surface of the cells (fig 1). The plasma membrane of microvilli may contain some of the enzymes and transporters known to be present in the apical parts of enterocytes. Such transporters have been proposed to function as sensors for the contents of the intestinal lumen. ${ }^{7}$

The two most thoroughly investigated endocrine cells of the small intestine are the cholecystokinin (CCK) and enterochromaffin (EC) cells. CCK cells are mainly located in the proximal part of the small intestine. The most potent stimulants of CCK secretion are digestion products of fat and protein. Furthermore, two types of luminal factors exist which are important for the secretion of CCK: a releasing factor produced by enterocytes and a monitor peptide, a releasing factor from the pancreas. Forskolin and CAMP analogues stimulate CCK release, possibly via a $\mathrm{Ca}^{2+}$ dependent mechanism tents. Na coupled solute transport of glucose, for
Abbreviations: CCK, cholecystokinin; EC enterochromaffin; $C A M P$, cyclic adenosine monophosphate; 5-HT, 5-hydroxytryptamine; CNS, central nervous system; NO, nitric oxide; NOS1, nitric oxide synthase 1 
(fig 1). Release of CCK is controlled by, among other things, adrenergic receptors. One type of CCK receptor (CCK-A receptor) has been localised to peripheral autonomic afferent neurones. ${ }^{8}$

EC cells are dispersed throughout the epithelium of the intestinal tract on villi and in crypts. In EC cells, granules containing 5-HT and various peptide hormones are concentrated around the basolateral surface of the cell. Stroking the intestinal mucosa evokes release of 5-HT from EC cells. As with CCK cells, increases in intracellular cAMP concentration stimulate 5-HT release via a $\mathrm{Ca}^{2+}$ dependent mechanism. ${ }^{9}$ Furthermore, release of 5 -HT/peptides is controlled by the autonomic nervous system. Stimulation of muscarinic receptors increases release while adrenergic stimulation inhibits release. $5-\mathrm{HT}_{3} / 5-\mathrm{HT}_{1 \mathrm{P}}$ receptors have been localised to afferent neurones in villi. It is interesting to note that the $5-\mathrm{HT}_{3}$ receptor is the only 5 -HT receptor known to be coupled to the opening of a cation channel, implying that activation of the receptor can influence membrane potential. It is obvious from the description above that luminal agents which induce release of amines/hormones from endocrine cells may, alone or together, activate nerves, provided there are appropriate receptors on afferent neurones in the vicinity of the endocrine cells. For example, considerable circumstantial evidence exists for the proposal that cholera toxin evokes net fluid secretion by stimulating the enteric nervous system, at least in part, via release of 5-HT from EC cells ${ }^{10}$.

It is well established that the sensitivity of the muscle spindles in skeletal muscle is controlled from the central nervous system (CNS) via efferent fibres influencing the socalled intrafusal muscle cells. One can envisage similar CNS control of the sensitivity of endocrine cells of the gastrointestinal epithelium via receptors known to be present on

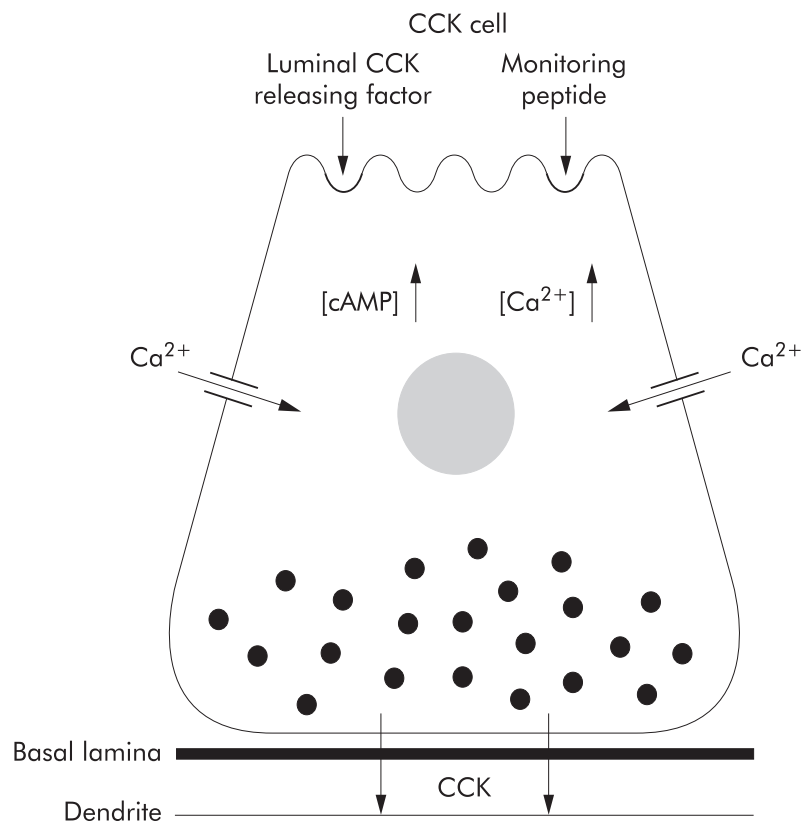

Figure 1 Schematic illustration of an intestinal endocrine cell containing cholecystokinin (CCK). Two luminal releasing factors are known: the luminal releasing factor of intestinal origin and the monitoring peptide of pancreatic origin. Activating their receptors release CCK from the basolateral cell membrane via mechanisms involving CAMP and $\mathrm{Ca}^{2+}$ ions. The details of these intracellular events are not yet fully elucidated. Plasma membrane $\mathrm{Ca}^{2+}$ channels are also involved in the release of CCK as it is possible to block its release with L-type calcium channel blockers. CCK-A receptors have been located on peripheral afferent neurones. endocrine cells. Activation of these receptors may change the sensitivity of endocrine cells to luminal stimuli by influencing their membrane potential.

\section{BRUSH CELLS}

There exists a specialised type of epithelial cell scattered within the epithelial lining of the respiratory and gastrointestinal tracts called a "brush cell", "tufted cell", or "caveolated cell". In the gastrointestinal tract, these cells have been found in the stomach and intestine, and also in the excretory ducts of the gall bladder and pancreas. They are pear or bottle shaped with a broad perinuclear portion located at the base (fig 2). An apical microvillus tuft is in contact with the intestinal lumen. No granules of the type present in endocrine cells can be demonstrated in brush cells. ${ }^{11}$

The functions of brush cells are unknown, although their morphology suggests a chemosensory function as they share distinct features with the taste receptor cells of the tongue. One recent observation suggests that brush cells may indeed have a chemosensory function. Brush cells of the rat stomach and intestine strongly react with antibodies to alphagustducin at the luminal pool of the cell. ${ }^{11}$ In taste receptor cells sensing bitter or sweet taste, gustducin is believed to couple putative receptor proteins of the plasma membrane to various $\mathrm{G}$ proteins. The question then arises: how may brush cells convey a message across the intestinal epithelium?

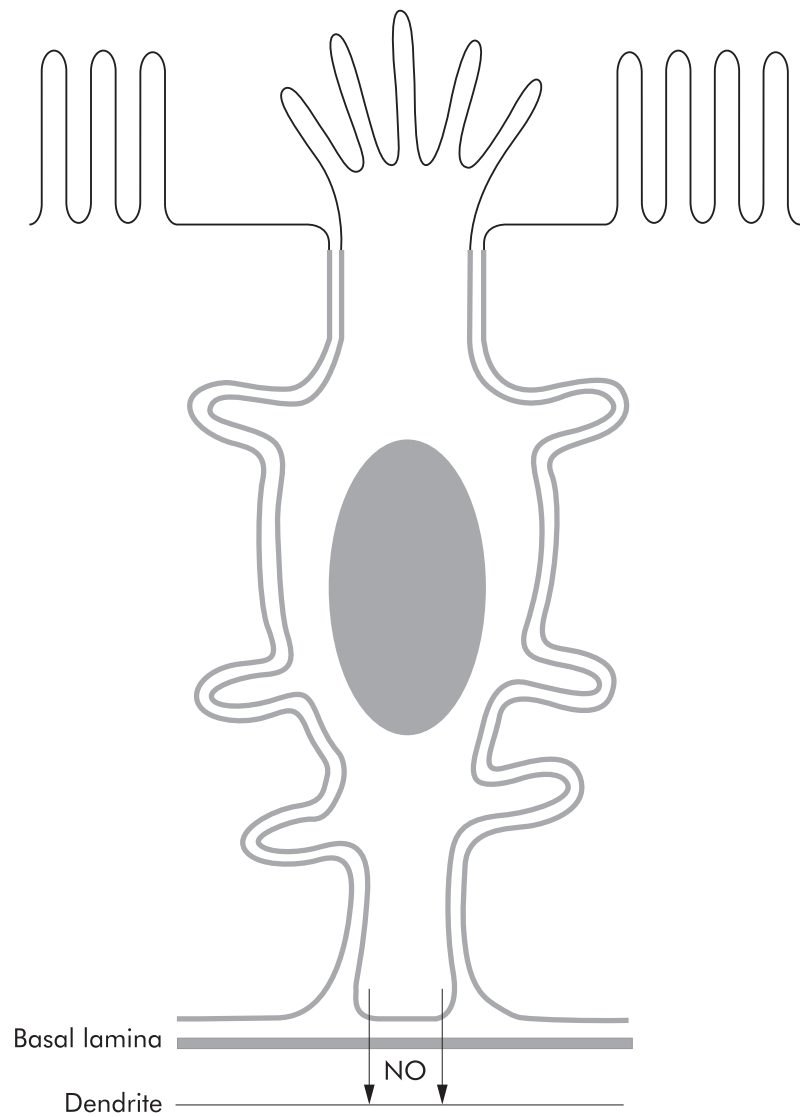

Figure 2 Schematic figure of a brush cell. The cell, located between enterocytes, has a bottle-like appearance. It lacks the granules at the basolateral part characteristic of endocrine cells (see fig 1). The fingerlike structures at the apical pole of the cell contain alpha-gustducin, also found in the taste buds of the tongue sensing sweet and bitter. A rich content of nitric oxide (NO) synthase suggest the possibility that the brush cells may release $\mathrm{NO}$ to activate nervous dendrites. CCK, cholecystokinin. 
Recent investigations indicate that nitric oxide (NO) may be an important molecule. Brush cells display an extremely strong immunoreactivity for NO synthase I (NOS I). ${ }^{11}$ Released NO may influence adjacent enterocytes or endocrine cells and may also directly activate nervous afferents. Thus it seems possible that luminal contents sensed by brush cells may activate villous afferent neurones via release of NO. However, such a mechanism remains to be demonstrated experimentally.

\section{ENTEROCYTES AS SENSORS FOR INTESTINAL MICROBES}

Mucosal surfaces of the gastrointestinal, respiratory, and genitourinary tracts represent outer surfaces exposed to the microbiological environment. They are therefore the most important routes of entry for microbial pathogens into the host and constitute important mechanical barriers separating the host's internal milieu from the external environment. During the past several years, experimental studies have indicated clearly that epithelial cells at mucosal surfaces play an integral role in generating and transmitting signals between invasive and non-invasive microbial pathogens and adjacent and underlying cells in the mucosa ${ }^{12}$.

Exposing intestinal epithelial cells to various strains of bacteria causes increased expression and secretion of a number of cytokines with chemoattractant and proinflammatory functions. Secreted cytokines are mainly of the type characterised by their ability to chemoattract and activate polymorphonuclear leucocytes. Expression and release of some interleukins are also increased by intestinal epithelial cells in response to microbes. Other biologically interesting substances to be released from enterocytes by luminal microbes are prostaglandins, NO, and intercellar adhesion molecule 1. The compounds released by enterocytes indicate that the products secreted by intestinal epithelial cells are likely to play an important role in the innate immunity response, rather than in the antigen specific mucosal immune response. However, there are also observations to suggest that enterocytes may participate in the antigen specific response as they are provided with MHC class II molecules and molecules of the CD 1 type located on the apical membrane of enterocytes. ${ }^{12}$

It is interesting to note that the response of enterocytes to luminal bacteria is more or less the same, regardless of the microbe involved. For example, Salmonella enters enterocytes, eventually to reach membrane bound vesicles, whereas Listeria rapidly lyses such vesicles to move freely within the cytoplasm. Yet the two types of microbe evoke a similar secretory response. It seems as though the enterocyte possesses a standardised secretory response when exposed to luminal microbes. Many of these compounds may, alone or together, activate afferent neurones in villi. ${ }^{13}$

\section{REFERENCES.}

1 Marcial MA, Carlson SL, Madara JL. Partitioning of paracellular conductance along the ileal crypt-villus axis: a hypothesis based on structural analysis with detailed consideration of tight junction structure-function relationships. $J$ Membr Biol 1984;80:59-70.

2 Fihn BM, Sjöqvist A, Jodal M. Permeability of the rat small intestinal epithelium along the villus-crypt axis: effects of glucose transport. Gastroenterology 2000;119:1029-36.

3 Weiser MM, Quill H. Intestinal villus and crypt cell responses to cholera toxin. Gastroenterology 1975;69:472-82.

4 Pappenheimer JR, Reiss KZ. Contribution of solvent drag through intercellular junctions to absorption of nutrients by the small intestine of the rat. J Membr Biol 1987;100:123-6.

5 Pappenheimer JR. Physiological regulation of transepithelial impedance in the intestinal mucosa of rats and hamsters. J Membr Biol 1987;100:137-48.

6 Madara JL, Pappenheimer JR. Structural basis for physiological regulation of paracellular pathways in intestinal epithelia. J Membr Biol 1987; 100:149-64.

7 Buchan AMJ. Nutrient tasting and signalling mechanisms in the gut. III. Endocrine cell recognition of luminal nutrients. Am J Physiol 1999;277:G1103-7.

8 Liddle RA. Cholecystokinin cells. Annu Rev Physiol 1997;59:221-42.

9 Timar Peregrin A, Ahlman H, Jodal M, et al. Effects of calcium channel blockade on intestinal fluid secretion: sites of action. Acta Physiol Scand 1997; 160:379-86.

10 Jodal $M$, Lundgren $O$. Neural reflex modulation of intestinal epithelial transport. In: Gaginella TS, ed. Regulatory mechanisms in gastrointestinal function. Boca Raton: CRC Press Inc, 1995:99-144.

11 Höfer D, Asan E, Drenckhahn D. Chemosensory perception in the gut. News Physiol Sci 1999;14:18-23.

12 Kagnoff MF, Eckmann L. Epithelial cells as sensors for microbial infection. $J$ Clin Invest 1997; 100:6-10.

13 Kirkup AJ, Brundsen AM, Grundy D. Receptors and transmission in the braingut axis: potential for novel therapies. I. Receptors on visceral afferents. Am J Physiol 2001;280:G787-94. 